\title{
IMPROVEMENTS TO THE PRETREATMENT OF BONE AT OXFORD
}

\author{
Christopher Bronk Ramsey ${ }^{1} \bullet$ Thomas Higham • Angela Bowles • Robert Hedges \\ Oxford Radiocarbon Accelerator Unit, University of Oxford, United Kingdom.
}

\begin{abstract}
Bone is one of the most widely used materials for dating archaeological activity. It is also relatively difficult to pretreat effectively and new methods are an area of active research. The purpose of the chemical pretreatment of bone is to remove contaminants present from burial and to do so in a way which does not add any additional laboratory contaminant. To some extent, these two aims must be balanced since, on the whole, the more complex the procedure and the more steps included, the greater the chance for contamination. At the Oxford Radiocarbon Accelerator Unit (ORAU), the method used is a continuous-flow or manual acid/base/acid (ABA) treatment followed by gelatinization and ultrafiltration (based on Brown et al. [1988]; documented in Bronk Ramsey et al. [2000]). We find this overall method is very effective at removing more recent contamination in old bones. However, two aspects of the method have recently been improved and are reported here: the redesign of ORAU's continuous flow pretreatment and a new protocol in our pretreatment ultrafiltration stage.
\end{abstract}

\section{INTRODUCTION}

Bone is one of the most complex sample materials routinely used in radiocarbon dating. The most effective method of pretreatment for accelerator mass spectrometry (AMS) dating has been a subject of continual research over more than $20 \mathrm{yr}$ since the method was first used. For many samples, a simple demineralization is effective at removing the most ubiquitous contaminants. An acid/base/ acid (ABA) treatment will also remove humic contaminants which can be significant in many organic rich contexts. However, these treatments leave many organic molecules from the soil and degraded protein fragments, making the sample susceptible to contamination.

Various further stages can be applied. Gelatinization (Law and Hedges 1989) concentrates the protein components, and ultrafiltration (Brown et al. 1988) selects large molecular weight proteins which are more likely to be from the original collagen present in the bone. In 2000, ORAU adopted the ultrafiltration method after detailed consultation with existing users of the method. This method in its original form was used for bone dates in the range OxA-9361 to -11851 and OxA-12214 to -12236. Tests on deliberately contaminated material showed that the method was more effective at removing contaminants, and results on known-age material were within expected limits.

The main elements of the current method are the following:

- Coarsely ground bone powder (about $0.5-1 \mathrm{~g}$ ) is loaded into a continuous-flow cell (Figure 1);

- An automated sequence of acid, base, acid is flowed through the cell over a period of $8 \mathrm{hr}$, rinsing with ultrapure (MilliQ ${ }^{\mathrm{TM}}$ ) water between each reagent (in some instances a manual version of this procedure is carried out in a test tube);

- Crude collagen is gelatinized in $\mathrm{pH} 3$ solution at $75^{\circ} \mathrm{C}$ for $20 \mathrm{hr}$;

- The gelatin solution is filtered using a $100 \mu \mathrm{m}$ polyethylene Eezi-filter ${ }^{\mathrm{TM}}$ and the insoluble residues discarded;

- The filtered gelatin is then pipetted into an ultrafilter (Vivaspin ${ }^{\mathrm{TM}} 1530 \mathrm{kD}$ MWCO) and centrifuged at $2500-3000 \mathrm{rpm}$ until $0.5-1 \mathrm{~mL}$ of the $>30-\mathrm{kD}$ gelatin fraction remains;

- This gelatin is freeze-dried and ready for combustion in a CHN analyzer.

${ }^{1}$ Corresponding author. Email: christopher.ramsey@archaeology-research.oxford.ac.uk.

(C) 2004 by the Arizona Board of Regents on behalf of the University of Arizona

Proceedings of the 18th International Radiocarbon Conference, edited by N Beavan Athfield and R J Sparks

RADIOCARBON, Vol 46, Nr 1, 2004, p 155-163 


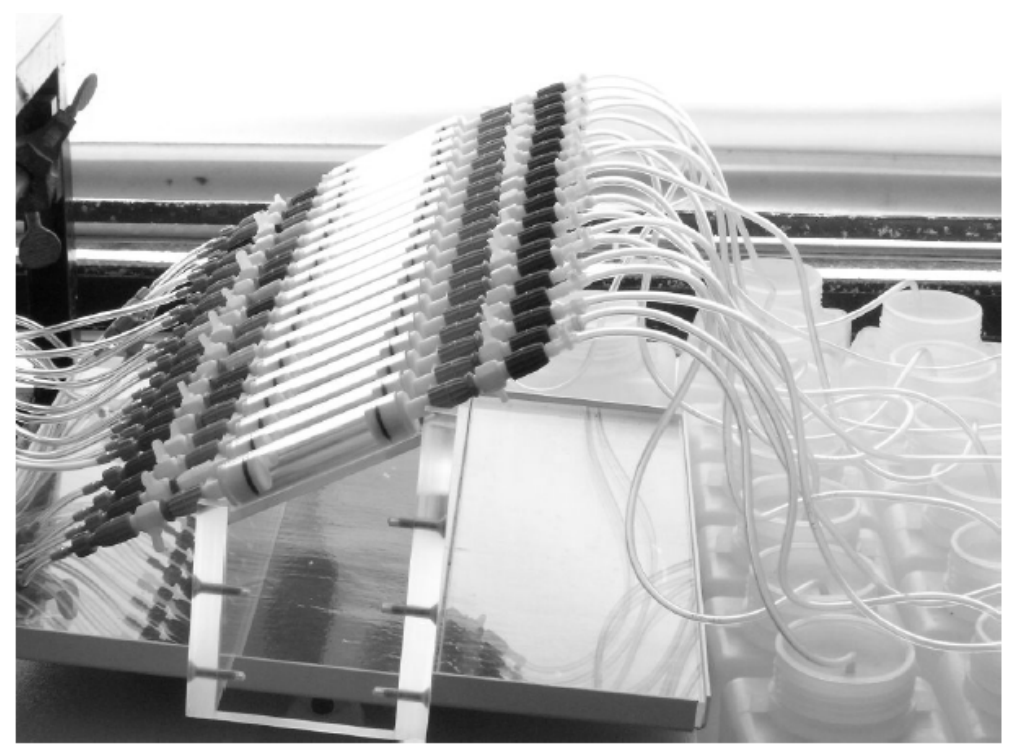

Figure 1 The continuous-flow system consists of an Ismatec IPC peristaltic pump, Omnifit ${ }^{\mathrm{TM}}$ connectors and tubing, shaking platform, glass vessels for acid/base/water, and glass flow cells. This enables semi-automated pretreatment of 20 samples of bone at once (Law and Hedges [1989] described the earlier version of what we now use).

Late in 2002, we became aware that some bone samples were giving ages which were about $100-300 \mathrm{yr}$ too old in context, and this included some bone of known age. This paper describes the investigation of this problem and the means through which it was resolved.

\section{PROBLEMS AND IMPROVEMENTS: ULTRAFILTRATION}

Monitoring of the processing contamination has always been undertaken by the routine measurement of background bone samples which are believed to be beyond the range of ${ }^{14} \mathrm{C}$. A summary of these measurements is shown in Figure 2. The data show that when we correct our bone collagen AMS measurements for known sources of contamination, there is no systematic effect with sample size, though arguably with the very oldest samples, at the lower limits of sample size, we may be underestimating our uncertainty. This is likely to be due to sample specific effects, but needs to be borne in mind in interpreting such results. There is no systematic effect with sample size, though there is slightly more variation than accounted for (about $0.1 \%$ average) and the results with yields $<5 \mathrm{mg}$ collagen are notably more scattered (Figure 2 ).

Measurements on background material do not, however, test for the presence of old contaminants in more recent samples and this was what we suspected in the case of the anomalous bone dates. We investigated possible sources of such contamination both in the original samples and in the laboratory process. Since no such systematic effect had been observed prior to the introduction of the ultrafiltration method, we suspected that the filters themselves might be responsible. The filters contain a humectant (glycerol) coated onto the regenerated cellulose filter membrane of the ultrafilters. This material is highly soluble in water and was expected to be of modern organic origin. The manufacturer's instructions are to rinse this out by centrifuging twice with ultrapure water. We had adopted the practice of centrifuging 3 times to ensure effective removal and monitoring the backgrounds as shown in Figure 2. 


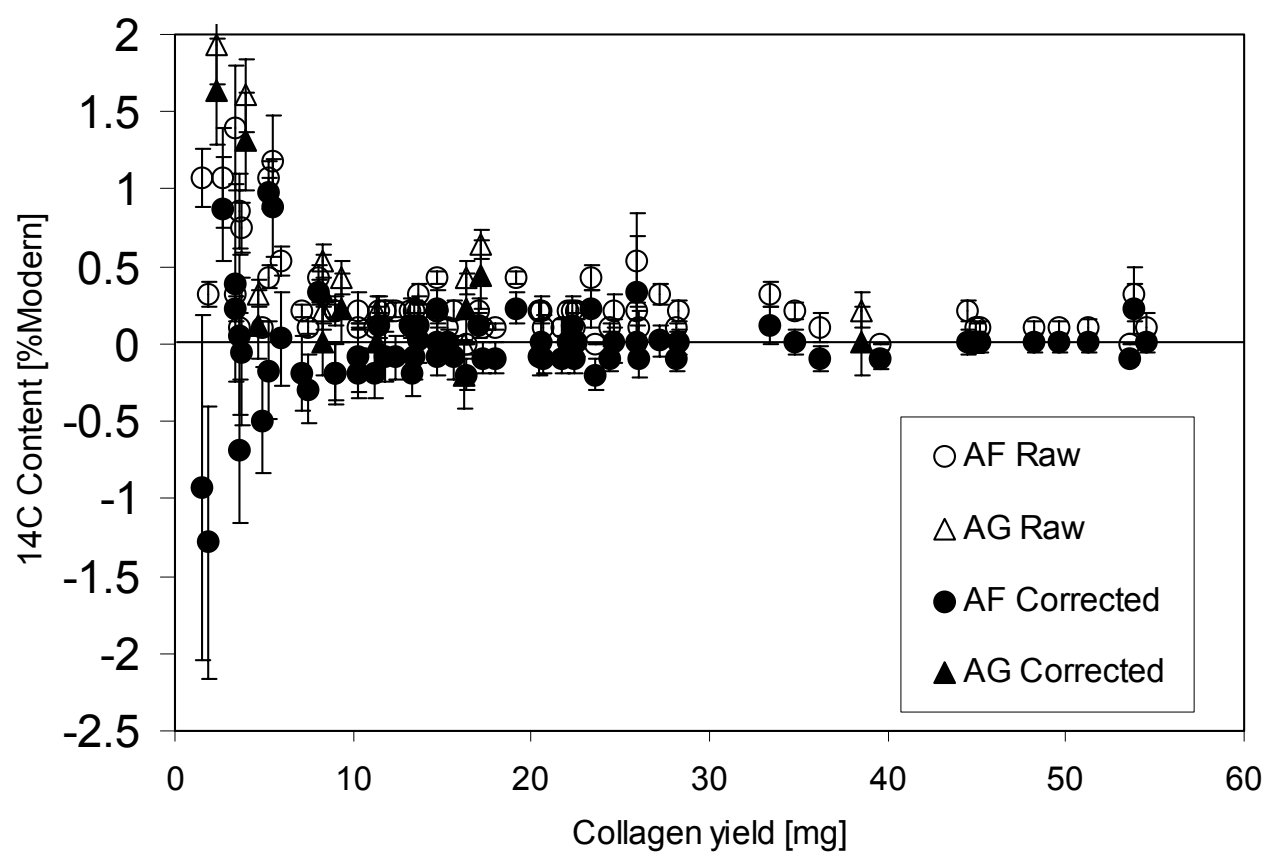

Figure 2 This figure shows a plot of ${ }^{14} \mathrm{C}$ concentration in relative percent modern as a function of collagen yield for measurements on a set of bones expected to be beyond the range of ${ }^{14} \mathrm{C}$. Two methods have been used for these measurements: acid/base/acid and gelatinization (AG) and the same method followed by ultrafiltration (AF). Raw results are as measured on the AMS and corrected results include corrections for known sources of background. The uncertainties are plotted at $2 \sigma$ and include estimates of quantified sources of background variation. Our normal cutoff for reliable measurements is 6-8 mg depending on starting weight. The scatter in pMC results for collagen yields $<5 \mathrm{mg}$ demonstrates the need for this acceptance threshold.

Because we suspected that the ultrafilters might be adding a fossil rather than an expected modern contaminant, we extracted and dated some glycerol from the filters and obtained a date of $>35 \mathrm{ka}$ BP. We devised a method for measuring the quantity of carbon remaining on the filters (most relevant in this method since the $>35 \mathrm{kD}$ molecular weight material remaining above the filter is pipetted off, lyophilized, and AMS dated) and in the eluent through the various phases of the ultrafilter cleaning by concentrating the glycerol onto Chromasorb pellets and combusting in a CHN analyzer. The results of these tests are shown in Figure 3 and were used to determine the most effective method of cleaning the ultrafilters prior to use. They show that the recommended method is clearly not sufficient, and the methods we applied to clean the filters also failed to remove all of the glycerol from the filters, despite its solubility in water. We found that 3 centrifuge rinses in ultrapure water left between approximately $20-50 \mu \mathrm{g} \mathrm{C}$, which could be retained above the filter along with the retained collagen. We found that if the filters are ultrasonicated prior to centrifugation, however, there was no measurable carbon remaining after the third centrifuge (Figure 3). The quantities of carbon left above the filters using the initial cleaning method we implemented are of the right order to explain the offsets we found (see below).

By ultrasonicating the filters in ultrapure water for $1 \mathrm{hr}$ after the first 2 rinses, then, the glycerol is much more effectively removed. Our method for the cleaning of the filters now comprises the following: 

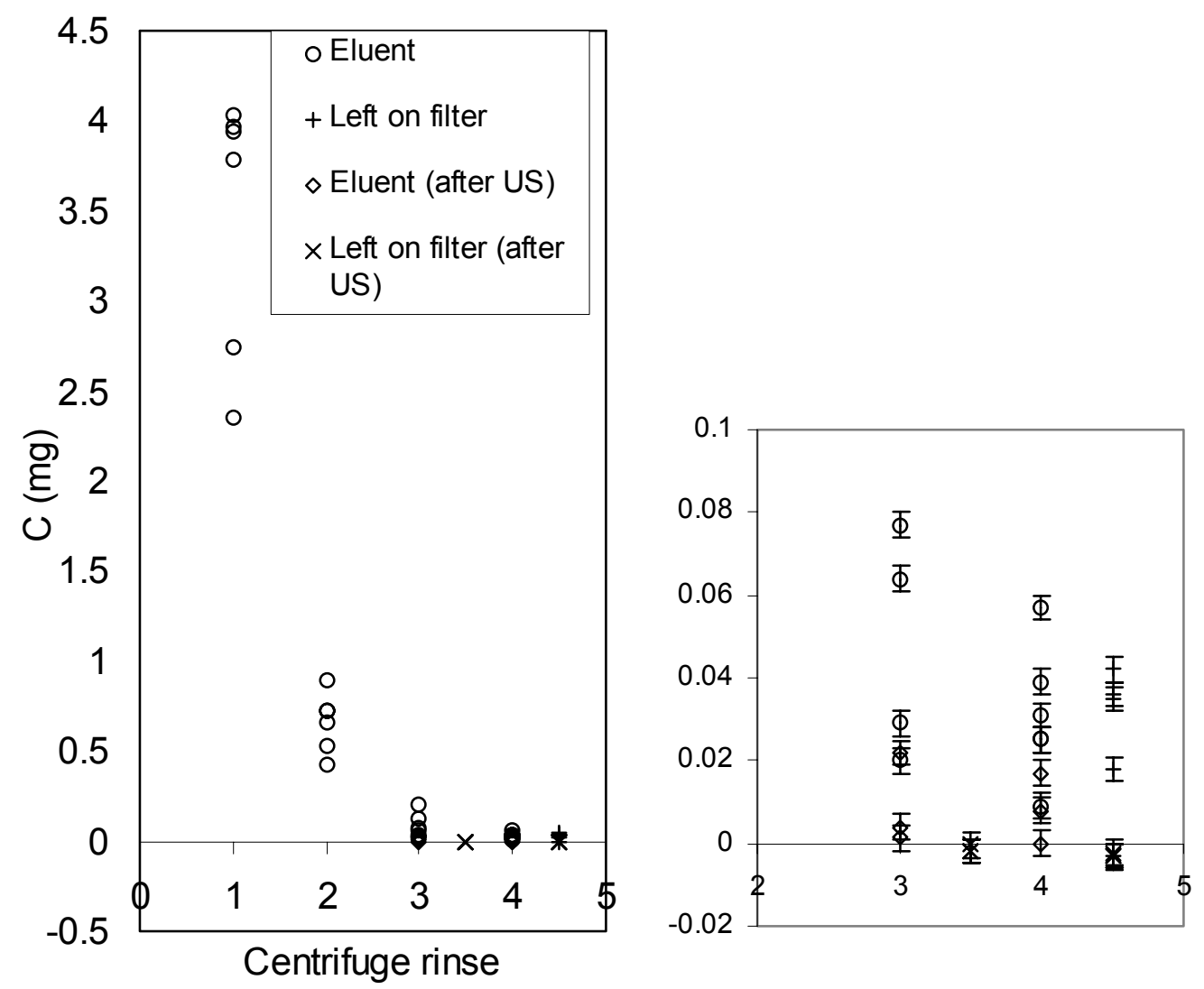

Figure 3 This figure shows the reduction in ultrafilter humectant during cleaning with centrifugation. In the cases marked "after US," the filters have been ultrasonicated in a large volume of ultrapure water for $1 \mathrm{hr}$ prior to the third centrifuge stage. In the bone pretreatment method, the eluent is discarded and the collagen above the filter is dated. The quantities remaining on the filter (that would be mixed with collagen) have been estimated by rinsing the top of the filter with ultrapure water and then concentrating this and measuring in a $\mathrm{CHN}$ analyzer. The figure on the right shows that 3 cleaning centrifuge rinses followed by the centrifuge of the collagen leaves about 20-50 $\mu \mathrm{g} \mathrm{C}$ mixed with the collagen. When the filters are ultrasonicated (diamonds and crossed symbols), there is no measurable carbon remaining after the third centrifuge.

- Two centrifuge rinses in ultrapure (MilliQ ${ }^{\mathrm{TM}}$ ) water;

- Ultrasonication in a large volume of ultrapure water;

- One centrifuge in ultrapure water;

- A sample is taken from the top of the filter for carbon content analysis (one for each batch of filters cleaned);

- Two further centrifuges in ultrapure water.

We found that bones we redated using the new filter cleaning protocol evidenced lower $\mathrm{C}: \mathrm{N}$ atomic ratios, which we correlate with the removal of a carbon-rich contaminant (Figure 4). In all cases, the original C:N ratios were well within acceptable limits (2.9-3.5 at ORAU) because the absolute proportion of contamination is very small. 


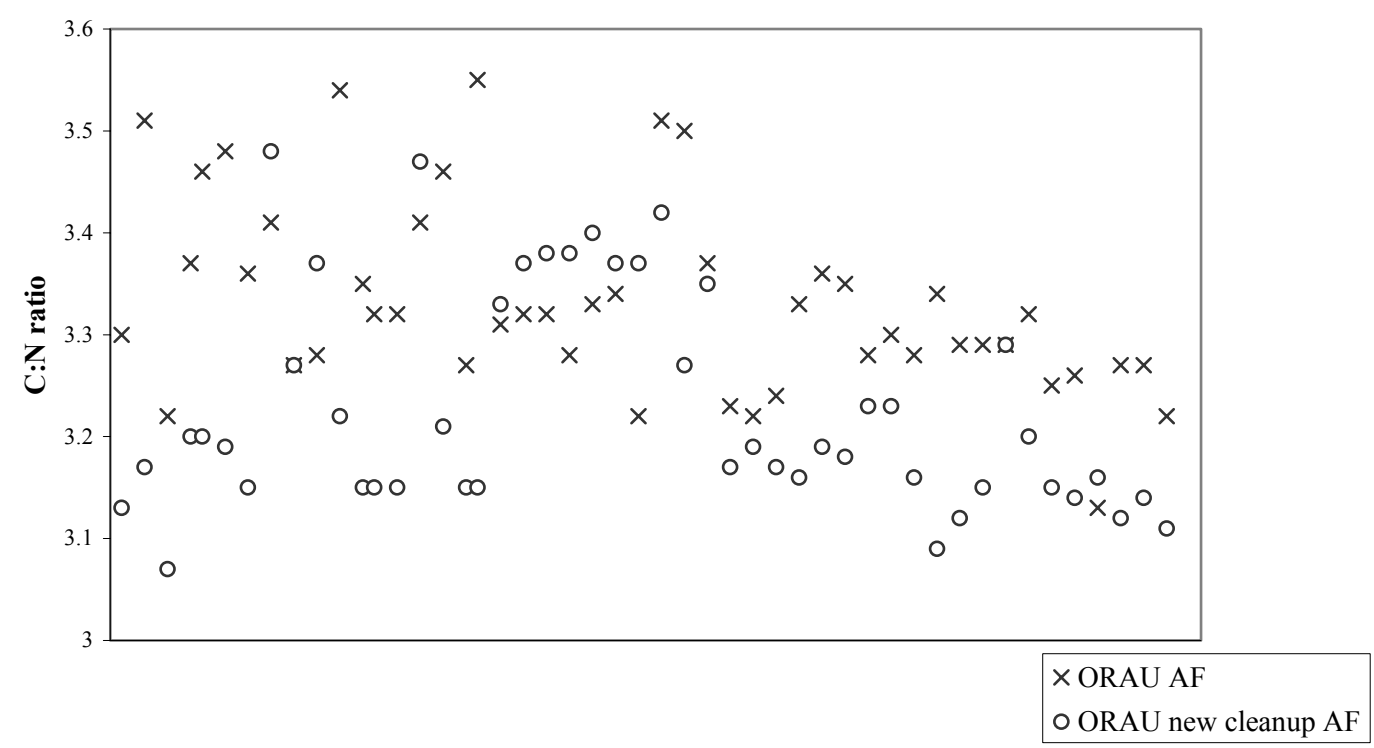

Figure $4 \mathrm{C}: \mathrm{N}$ data before and after the introduction of the new cleanup method: There is a shift in C:N ratios which occurs when original bone, dated using our ultrafiltration method, is redated using our new cleanup method for the ultrafilters. Since the humectant used in the filters has no nitrogen, the higher C:Ns in the original AF series indicate the humectant was not effectively removed.

\section{CONTINUOUS-FLOW SYSTEM}

The lower than expected yields were in some cases found to be linked to the use of the semi-automated continuous-flow system which is usually used, since the steady flow of fresh reagents is more effective at removing contaminants present in the original sample. We think that the low yields were occurring when the continuous-flow cells blocked (particularly likely in the case of fine bone powder). In these instances, the bones were subjected to the base solution for longer than intended, degrading the collagen.

We have now redesigned and constructed a new continuous-flow system. We installed an Ismatec ${ }^{\mathrm{TM}}$ IPC peristaltic pump and replaced the flowcells described in Law and Hedges (1989) with variable length $(50-100 \mathrm{~mm})$ preparative chromatography borosilicate glass columns fitted with Omnifit ${ }^{\mathrm{TM}}$ PTFE endpieces, caps, and viton O-rings. We use 100- $\mu \mathrm{m}$ PTFE frits at both ends of the glass columns. We also replaced all tubing and connectors from the peristaltic pump to the glass columns and to waste, with Tygon ${ }^{\mathrm{TM}}$ tubing and Omnifit ${ }^{\mathrm{TM}}$ Peek barbed adapters (Figure 1). The glass columns sit on a shaking platform which reduces blockage and mixes reagents more effectively. This system is much less prone to blocking and eliminates leaking from the flow cells.

\section{RESULTS}

One important means of testing the reliability of a method is to monitor process blanks as described above. However, the most effective test is to make measurements on independently dated, knownage material. In the case of bone, this is not straightforward, because dietary effects and reservoir corrections account for offsets from the true age for some types of bone, particularly omnivorous species. The bones we selected are a set of pig bones recovered from the wreck of Henry VIII's flagship, the Mary Rose, which sank in AD 1545. Known-age bones of this sort are usually well preserved since they are usually from historic periods. Mary Rose bones yield routinely 130-150 mg 
collagen/g, so in order to simulate low collagen yields, we have dated a series of samples using lower starting weights than usual.

The results of measurements of the Mary Rose bones are shown in Figure 5. All were measured on the new ORAU AMS, which began routine measurements in September 2002 (Bronk Ramsey et al., these proceedings). These show a very good level of agreement - certainly until the yields drop to well below $10 \mathrm{mg}$. The subset of measurements analyzed using the new filter cleaning method (denoted by $\mathrm{AF} / \mathrm{AF}^{*}$ new) shows that for collagen yields $>7 \mathrm{mg}$ (13 measurements), the average ${ }^{14} \mathrm{C}$ result is $321 \pm 6.5 \mathrm{BP}$, with a standard deviation of $29.9 \mathrm{yr}$ and an average precision of $23.7 \mathrm{yr}$ [this just passes a $\chi^{2}$ test $\left(T=19.5 ; \chi^{2} 1: 0.05=21.0\right)$; the combined result also passes a $\chi^{2}$ test against $309 \pm 4.6 \mathrm{BP}$, the INTCAL98 value for AD $\left.1545\left(T=2.27 ; \chi^{2} 1: 0.05=3.84\right)\right]$. None of the measurements using the original ultrafiltration cleaning method have a yield of $<25 \mathrm{mg}$ collagen and, notably, all of these measurements are in agreement with the expected value.

a)

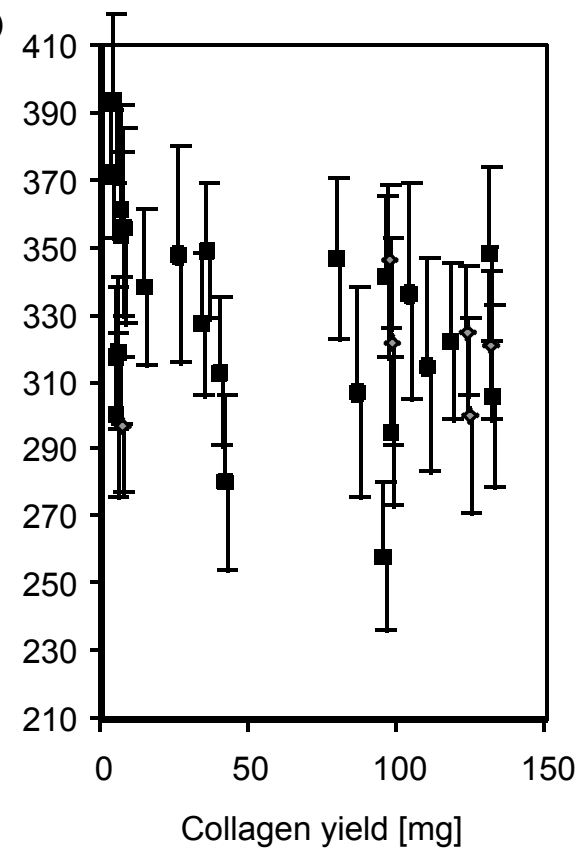

b)

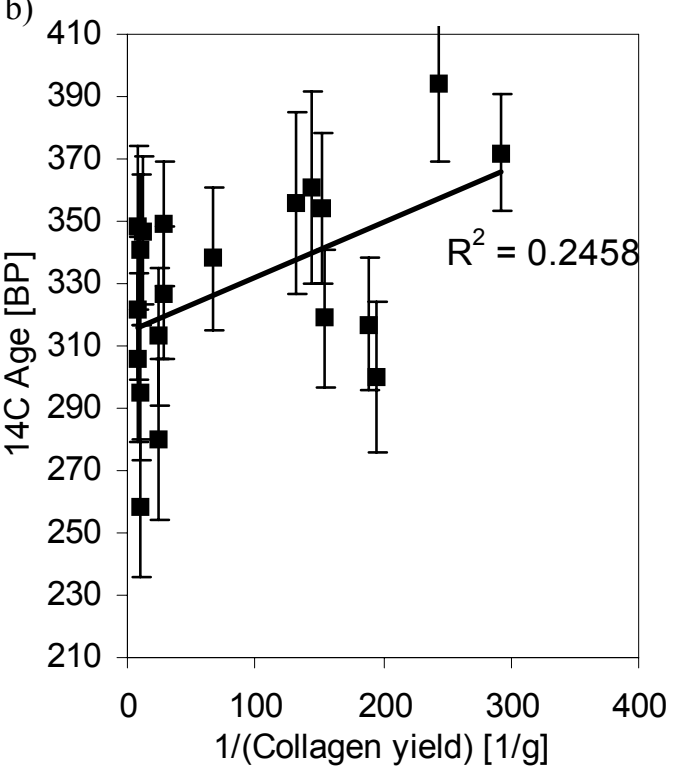

Figure 5 Results from pig bones found on the wreck of the Mary Rose (sank in AD 1545, so should be about 310 BP). a) All dates measured on our new AMS are shown here. AG - denotes gelatin pretreatment; AF - denotes ultrafiltration, old - with the old cleanup method and new - with the new cleanup of the ultrafilters. * - denotes solvent extraction. They are virtually indistinguishable (sigma 31 , average uncertainty 27 ).

b) The regression line of date versus reciprocal yield, with the intercept exactly in accord with the historical date. An increase of age with reciprocal yield suggests the possible presence of low levels of an older contaminant unrelated to bone sample size, although at a level that is not significant for the normal sample size range. The $\mathrm{R}^{2}$ value is also low (0.2458), so we cannot be sure of the significance of the sample size effect. See main text for discussion

A linear regression of the AMS determinations versus reciprocal yield (Figure 5) for the ultrafiltration method using the new cleaning protocol indicates a small residual effect equivalent to fossil carbon contamination at the $5-10 \mu \mathrm{g}$ level. This would produce a ${ }^{14} \mathrm{C}$ result too old by about $15-20 \mathrm{yr}$ for samples with a yield of $<10 \mathrm{mg}$ (this encompasses a very small proportion of bone samples where the original sample is low in collagen content and poorly preserved due to post-depositional 
burial conditions). It is not clear that this effect (constant amount of old carbon) is laboratoryderived, although it may well not originate from the ultrafiltration method itself. Generally speaking, the data show it to be reduced to an acceptable level for high-precision dating of bone with the normally accepted range of yields, though a bias of about $10 \mathrm{yr}$ cannot be ruled out. For very lowyield bones, further research is needed before this method can be used in high-precision work.

\section{Measurements of the Effectiveness of Ultrafiltration}

Given that the ultrafiltration method involves a significant amount of technician time to ensure that the filters are effectively cleaned, one might question whether it is strictly necessary for routine, reliable dating. We have some evidence which suggests that ultrafiltration is advantageous in some instances and produces more accurate ages than less rigorous bone pretreatment methods. We redated a series of samples which were originally pretreated using the gelatinization method without ultrafiltration (ORAU code AG) and which gave anomalous ${ }^{14} \mathrm{C}$ results within their archaeological contexts. The ultrafiltered collagen samples produced AMS results in much closer agreement with other determinations. We have also tested the effect of ultrafiltration on previously determined AG pretreated samples by ultrafiltering and dating a sub-sample of collagen remaining from the original pretreatment (Table 1). The results suggest strongly that the additional ultrafiltration pretreatment does have a significant effect.

Table 1 Comparison of bone originally dated after simple gelatinization with new dates pretreated using ultrafiltration of an aliquot of the original gelatin. The changes are significant. In one case, the filtration seems to have removed a younger contaminant and in the other 3 cases has removed an older contaminant. In the case of the samples from Ecsegfalva, the results are now in better agreement with other dates from that site in related contexts and are in very good agreement with each other. Note that these results are measured using the new ultrafiltration protocol, so we are confident the differences are not because of added contaminant.

\begin{tabular}{llllc}
\hline Site & \multicolumn{2}{l}{$\begin{array}{l}\text { Original AMS result (gelatin, } \\
\text { AG pretreatment code) }\end{array}$} & \multicolumn{2}{l}{$\begin{array}{l}\text { New AMS result (ultrafiltered gelatin, } \\
\text { AF pretreatment code) }\end{array}$} \\
\hline $\begin{array}{l}\text { German } \\
\text { Upper-Paleolithic }\end{array}$ & OxA-11964 & $23,990 \pm 180$ & OxA-12057 & $25,050 \pm 320$ \\
Ecsegfalva & OxA-9329 & $6950 \pm 45$ & OxA-X-2040-09 & $6780 \pm 45$ \\
Ecsegfalva & OxA-9327 & $6870 \pm 50$ & OxA-X-2040-07 & $6787 \pm 37$ \\
Ecsegfalva & OxA-9526 & $6915 \pm 50$ & OxA-X-2040-08 & $6775 \pm 37$ \\
\hline
\end{tabular}

More evidence is provided when we consider AMS determinations we obtained on a series of mammoth bones, performed in an inter-laboratory comparison with a conventional ${ }^{14} \mathrm{C}$ laboratory which used a modified Longin pretreatment method (Table 2). This shows that, particularly for older dates, the ultrafiltration method provides an improved removal of contaminants in the dated bones as evidenced by the older results. The implication of this is that, even for younger bones, if high levels of precision are required, ultrafiltration enables us to make a more reliable measurement on the uncontaminated collagen. To put this in context, the average offsets shown in Table 2, if due to modern contamination, would result in age offsets of about $1 \%$ (e.g. $40{ }^{14} \mathrm{C}$ yr at $4000 \mathrm{BP}$ ) for samples less than 1 half-life old. In many instances, particularly for recent, well-preserved bone, there is little doubt that the contaminants present are probably sufficiently close in age to the original material that even the simplest pretreatment methods will provide accurate ${ }^{14} \mathrm{C}$ results. 
Table 2 A comparison of ultrafiltered ORAU ${ }^{14} \mathrm{C}$ determinations (original method) on mammoth bone compared with those obtained at an anonymous conventional laboratory using a more standard bone pretreatment method. The differences are in some cases greater than $1000 \mathrm{yr}$, far larger than any offsets seen from addition of humectant, and in these cases the bones give high yields and are relatively old, so the differences must arise from the removal of contaminant by the ultrafiltration.

\begin{tabular}{llcc}
\hline $\begin{array}{l}\text { Conventional laboratory }{ }^{14} \mathrm{C} \text { age BP } \\
\text { using modified Longin method) }\end{array}$ & $\begin{array}{l}\text { Ultrafiltered gelatin AMS }{ }^{14} \mathrm{C} \text { age } \\
\text { (ORAU) }\end{array}$ & $\begin{array}{l}\text { Collagen yield } \\
(\mathrm{mg})(\mathrm{ORAU})\end{array}$ \\
\hline $12,500 \pm 50$ & OxA-11840 & $13,180 \pm 60$ & 76.1 \\
$13,200 \pm 100$ & OxA-11749 & $14,165 \pm 65$ & 10.7 \\
$17,450 \pm 100$ & OxA-11842 & $17,740 \pm 90$ & 170.0 \\
$28,600 \pm 600$ & OxA-11813 & $30,420 \pm 250$ & 17.0 \\
$42,600 \pm 1000$ & OxA-11747 & $45,200 \pm 800$ & 37.0 \\
$23,800 \pm 400$ & OxA-11746 & $23,560 \pm 140$ & 21.8 \\
$35,600 \pm 400$ & OxA-11748 & $36,610 \pm 360$ & 39.8 \\
\hline
\end{tabular}

\section{CONCLUSIONS}

The data presented in this paper suggest that the ultrafiltration method as originally devised (Brown et al. 1988) and implemented at ORAU (documented in Bronk Ramsey et al. 2000) was a considerable advance in terms of removing environmental contamination and was the best method available for older bones. When isolating collagen with low mass yields and recent ages, however, significant bias to older ${ }^{14} \mathrm{C}$ ages has been shown to arise and, in these cases, the results are usually less accurate than when a simpler method is used, because environmental contamination is less of an issue with these samples.

We contacted the submitters of samples with low collagen yields when we had identified the relationship between low pretreatment yield and older than expected AMS determinations. We are in the process of repeating measurements where spare or replacement bone could be obtained and we withdrew some of the original determinations that could not be repeated. We also offered recalculated ${ }^{14} \mathrm{C}$ ages to some submitters based upon the collagen pretreatment yield and the proportion of admixed humectant carbon derived from the ultrafilters. These recalculations (given an OxA-X lab code which indicates a non-standard date calculation) will only give an indication of the scale of the effect given the variability we have subsequently seen. In a few cases, the repeated measurements even on low-yield samples were unchanged; in other cases, there were changes consistent with the effects identified through our experimental data, although the exact scale of the offsets seems to be variable and sometimes larger than expected. One such significantly affected example is shown in Figure 6. This demonstrates the age shifts between the initial series affected by ultrafiltration humectants and the redated series, which ought to have a "true age" of AD 873/4 or 1190-1205 BP. When our current re-measurement program is finished, we should be in a better position to estimate the effect on intermediate collagen yield samples, but at present it looks as if the effect could average of the order of a hundred years until collagen yields rise above about $40 \mathrm{mg}$.

The key improvements to the new protocol at Oxford are in the preparation of the ultrafilters used in the final stage of the pretreatment and in the design of the continuous-flow system used for isolating bone collagen. The method has been shown to be effective both in providing good reproducibility and accuracy on recent known-age material and in removing environmental contaminants. We have also introduced regular process tests to identify contaminants from single batches of ultrafilters prior to routine use, and implemented QA checks on known-age recent bones over a range of sizes to test for any other similar effects. 


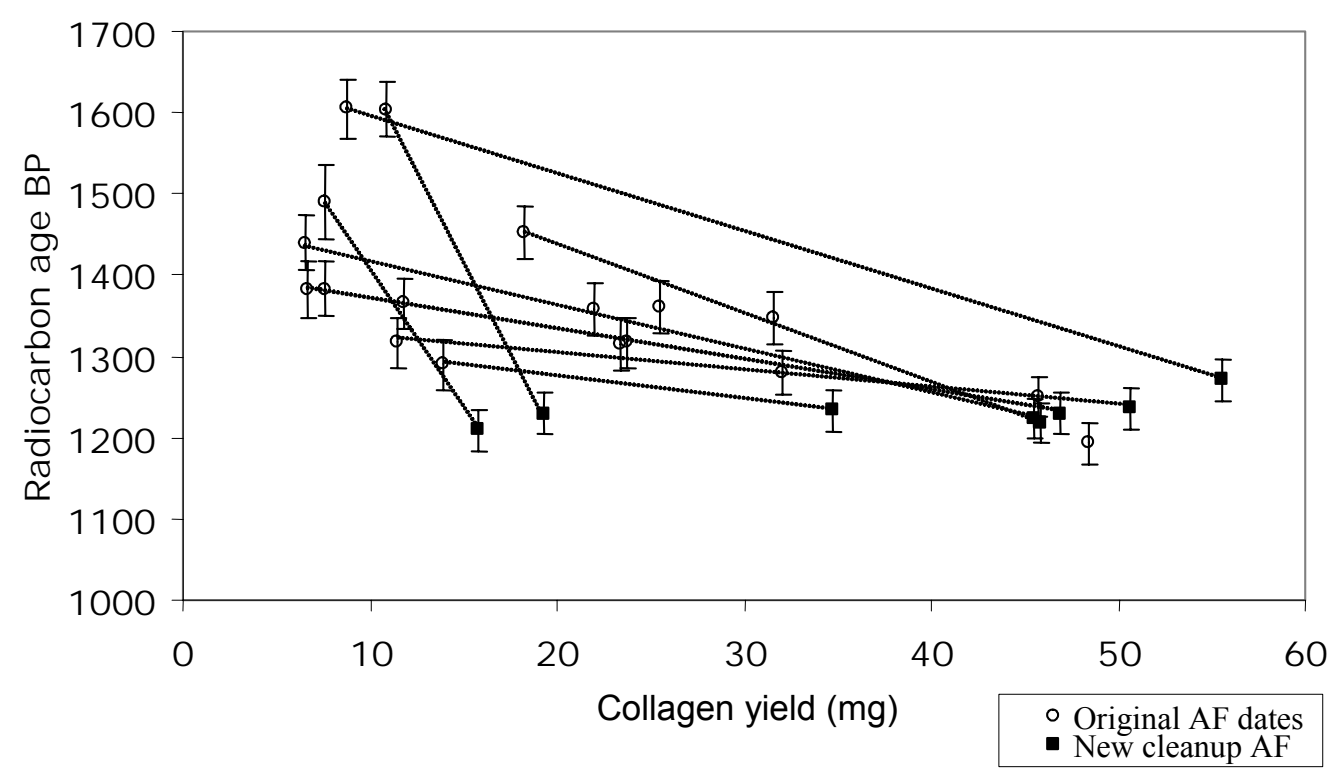

Figure 6 The Repton Viking Army site (Derbyshire, England), historically dated to AD 873/4 (1190-1205 BP). The initial humectant-affected dates are shown in open circles. The black squares are later determinations obtained with the new filter cleanup procedure. Even in this fairly severely affected batch, the higher yield dates are clearly closer to the true values.

During this research, we also conducted tests on other filter types used in ${ }^{14} \mathrm{C}$ dating and although we found that some gave no measurable additions (e.g. the Eezi-filter ${ }^{\mathrm{TM}}$ we use for removing particulate contaminants), others did have extractable carbon, although only ultrafilters had levels as high as those reported here. As ${ }^{14} \mathrm{C}$ sample requirements for AMS become smaller, this is something all ${ }^{14} \mathrm{C}$ labs will need to be aware of, and monitor.

\section{ACKNOWLEDGEMENTS}

The staff of ORAU are thanked for their involvement in all aspects of this work. We would also particularly like to thank Alex Bayliss and Peter Marshall at English Heritage for bringing this issue to our attention as well as English Heritage and NERC for funding. We are grateful to Dr A J Stuart (UCL), Professor M Biddle and B Kjolbye-Biddle (Oxford), Dr J Grunberg (Landesmuseum fur Vorgeschichte, Halle), and Professor A Whittle (University of Wales, Cardiff) for permission to publish data associated with their projects. We thank Dr Margaret Rule and Mr Andrew Elkerton (Mary Rose Trust) for providing the pig bones from the Mary Rose.

\section{REFERENCES}

Bronk Ramsey C, Higham TFG, Leach P. 2004. Towards high-precision AMS: progress and limitations. Radiocarbon, these proceedings.

Bronk Ramsey C, Pettitt PB, Hedges REM, Hodgins GWL, Owen DC. 2000. Radiocarbon dates from the Oxford AMS system: Archaeometry datelist 30. Archaeometry 42(2):459-79.
Brown TA, Nelson DE, Vogel JS, Southon JR. 1988. Improved collagen extraction by modified Longin method. Radiocarbon 30(2):171-7.

Law IA, Hedges REM. 1989. A semi-automated bone pretreatment system and the pretreatment of older and contaminated samples. Radiocarbon 31(3):247-53. 